\title{
Child and Family Predictors of Child-Teacher Relationship Trajectories during the Transition from Preschool to School
}

\author{
Lauren R. Miller-Lewis, Alyssa C. P. Sawyer, Amelia K. Searle, and Michael G. Sawyer
}

\begin{abstract}
This longitudinal study aimed to identify child, socio-demographic, family, and relationship factors associated with children's teacher relationship quality trajectories over the transition from preschool into school. A community sample of 636 Australian children were assessed in preschool, the first year, and second year of formal schooling. Teachers at all three assessments reported on child-teacher relationship quality. At preschool, teachers and parents reported on children's mental health difficulties and prosocial skills, and parents reported on family socio-demographic characteristics, stress, parenting styles, and parent-teacher relationships. Findings from latent-class growth modeling identified a 1) stable-high and 2) a moderate/declining child-teacher relationship trajectory. Logistic regressions found the strongest predictor of belonging to the moderate/declining relationship trajectory was the presence of teacher-rated preschool mental health difficulties. Children exposed to hostile-ineffective parenting were also more likely to experience a moderate/declining trajectory, whereas girls and children with more prosocial skills at preschool were less likely to have a moderate/declining trajectory. For children considered at-risk at preschool due to early mental health difficulties, prosocial skills and parental warmth appeared to protect them from the moderate/declining child-teacher relationship trajectory. Findings suggest that early-interventions focusing on fostering prosocial skills and positive parenting styles may help improve child-teacher relationships over time.
\end{abstract}

Index Terms-Child-teacher relationship trajectories, prosocial skills, mental health, family characteristics, young children.

\section{INTRODUCTION}

\section{A. Child-Teacher Relationships}

Teachers play a valuable role in the lives of young children. Following their parents, teachers are usually the adults that spend the greatest amount of time with children, and in the early years children are quite dependent on teachers for

Manuscript received July 25, 2013; revised November 18, 2013. This work was supported in part by funding from the National Health and Medical Research Council (Grant: 399225), Channel 7 Children's Research Foundation South Australia, Australian Rotary Health, and University of Adelaide's Faculty of Health Sciences.

Lauren R. Miller-Lewis and Michael G. Sawyer are with the Discipline of Paediatrics, School of Paediatrics and Reproductive Health, University of Adelaide; and the Research and Evaluation Unit, Women's and Children's Health Network, Australia (e-mail: lauren.millerlewis@adelaide.edu.au; michael.sawyer@adelaide.edu.au).

Alyssa C. P. Sawyer is with the Discipline of Public Health, School of Population Health, University of Adelaide, Australia (e-mail: alyssa.sawyer@adelaide.edu.au).

Amelia K. Searle is with the Centre for Traumatic Stress Studies, School of Population Health, University of Adelaide, Australia (e-mail: amelia.searle@adelaide.edu.au). guidance and emotional support, with teachers serving a similar function to a caregiver [1].

Therefore, caring and supportive child-teacher relationships may serve important functions for children's wellbeing and their successful adaptation to the school environment, and may be particularly salient at critical periods such as the transition from preschool into formal schooling [2], [3]

Developmental contextual systems models propose that children develop within the family and school systems and the key mechanisms through which they acquire developmental competencies are the dynamic relationships they form with parents and teachers [3]-[5]. Within these models, the quality of child-teacher relationships over time is the result of various factors within family and school contexts and within the child. Children's characteristics may contribute to stability in child-teacher relationships, whereas changes in family or school contexts may contribute to changes in relationship quality [6].

There is considerable evidence that supportive relationships between children and teachers facilitate healthy child development (see [3] and [4] for reviews). High quality child-teacher relationships characterized by close supportive interactions and low conflict contribute to children's academic development, engagement in learning, social-emotional development, and mental health (e.g., [1], [2], [7]-[11]). As such, early interventions designed to improve the quality of child-teacher relationships have the potential to improve the psychosocial and educational development of children [3].

Information about factors which influence the quality of child-teacher relationships is an important prerequisite to the development of new interventions in this area. However, to date, relatively little is known about the child, family, and school factors which influence the quality of child-teacher relationships [5].

Previous research has found several social and demographic factors associated with child-teacher relationships. Boys tend to have poorer quality relationships with teachers than girls [7], [8], and children with disabilities often experience poorer quality relationships with teachers [12]. Children with better quality relationships with their parents (including more secure attachments) tend to have better quality relationships with their teachers [12]-[15] Children whose parents have more positive and more involved relationships with their child's teachers have also been found to have better quality child-teacher relationships [8], [16]. Studies have also found associations between factors representing socioeconomic disadvantage (e.g., low maternal education, low family income and poverty) and 
poorer quality child-teacher relationships [2], [17]-[19]. Children with more prosocial behavior skills have also been found to experience better quality relationships with teachers, including more closeness and less conflict [7], [13].

Of all factors previously examined, there is the most substantial evidence implicating early mental health problems as influential on children's quality of relationships with teachers. In a meta-analysis of 19 studies, [20] found that when teachers interact with children exhibiting a high level of externalizing or internalizing problems, teachers report more conflict and less closeness in the child-teacher relationship. There is also longitudinal evidence to suggest that positive child-teacher relationships may be particularly important for healthy developmental outcomes in children at-risk due to exhibiting early behavioral problems, e.g., [21], [22]. Furthermore, there is indication that behavior problems and child-teacher relationships are part of a transactional cycle, whereby early aggressive behavior problems are related to an increase in child-teacher conflicts during the year, which is also related to an increase in behavior problems by the end of the year [23].

\section{B. Child-Teacher Relationship Trajectories}

Most studies examining child-teacher relationships have assessed the quality of these relationships at a single point in time. Such assessments provide no information about whether the quality of the relationships being assessed are improving, declining, or remaining stable. This is important because repeated and cumulative experiences of relationship difficulties with teachers over time may have a greater and more lasting impact on children's development than temporary or episodic difficulties experienced with a single teacher over a short period of time [6].

There are few studies that have examined the developmental trajectories of child-teacher relationships across the early school years [5]. Evidence suggests that teacher-reports of relationship quality with children are relatively stable across the early schooling years. For example, [14] found positive correlations in children's relationship quality with teachers in the first years of school, and that relationship quality with teachers at age 4 more strongly predicted kindergarten and first grade teacher-child relationships than maternal attachment. Nonetheless, some variation does exist in child-teacher relationship quality across the early school years, with moderate year-to-year stability usually found [24]. Another study [10] found only moderate stability in the quality of children's relationships with their different teachers in the first three years of school, demonstrating the dynamic and dyadic nature of relationships between a child and their various teachers. Subgroups of children who followed different growth trajectories for child-teacher relationship from preschool to third grade were then identified. While most children experienced moderate to good relationships with their teachers, they found a subgroup of children (13\%) who had poor relationships throughout early school and experienced declining relationship quality from year to year.

A small number of recent studies have produced emerging evidence that children's cumulative experiences in their relationships with teachers over time have important implications for psychological and academic development [25]. Longitudinal studies of elementary school children have found that positive trajectories of child-teacher relationship quality (characterized by children experiencing high closeness and less conflict over time) are significantly associated with better academic outcomes [6], [10], and better mental health outcomes [18], [25], [26]. For example, [25] used person-centered longitudinal analyses to identify different trajectory groups on teacher-child relational closeness and conflict in the first 5 years of school. They found that children in groups with elevated levels of conflict demonstrated higher levels of externalizing behaviors than those in the low-conflict group, and children in the stable-low-closeness group had higher levels of internalizing behaviors than those in the high-closeness group. In another study, [10] found that over the first three years of school, a small decrease each year in child-teacher relationship quality was predictive of lower levels of achievement by third grade.

O'Connor [5] states that examining factors associated with changes in child-teacher relationship quality is important, because even small changes in quality can have implications for child developmental outcomes. Accordingly, [5] is one of only two studies we are aware of that have extensively examined predictors of child-teacher relationship trajectories. This study investigated child, family, teacher, and school predictors of the quality of child-teacher relationships from first to fifth grade at elementary school in the United States NICHD cohort. O'Connor [5] found that children whose parents had more and higher quality interactions with the school had higher quality child-teacher relationships in fifth grade, and showed less rapid rates of decline in child-teacher relationship quality. Children in schools where teachers received higher salaries and better-managed classrooms that had more positive emotional climates evidenced slower rates of decline in relationship quality. While family income, maternal education, maternal attachment, and child temperament were not significantly related to child-teacher relationship trajectories, [5] found that girls, European-American children, children with fewer behavior problems, and children who had better relationships with their kindergarten teachers, all had higher quality relationships with teachers in fifth grade. O'Connor [5] concluded that children's relationships with kindergarten teachers are extremely important because they appear to form a basis for future relationships. Other studies by this group using person-centered approaches capable of detecting subgroups of children that follow atypical trajectories did find that children with an early insecure-other (disorganized) maternal attachment were more likely than others to have a declining trajectory of child-teacher relationship quality between pre-kindergarten and third grade [10], and specifically were more likely to show stable low-closeness in their child-teacher relationships [25].

The other major study investigating characteristics predicting differences in growth trajectories in child-teacher relationship also used the NICHD cohort data, from kindergarten through sixth grade [24]. They found child-teacher conflict was higher for children who were male, African-American, had spent more time in childcare, had mothers with less education, had poorer quality home environments, and had greater externalizing and internalizing behavior at age 4.5. Importantly, children with less sensitive mothers were at greater risk for increased conflict with 
teachers over time. Girls, children with higher quality home environments, mothers holding more educational experience, and mothers who showed greater sensitivity at age 4.5 had higher levels of teacher-reported closeness over time.

Some additional evidence comes from a study examining the influence of child-teacher relationship trajectories on academic outcomes in elementary school [6]. This study found that boys, economically disadvantaged children, children with lower intelligence, early behavior problems, and African-American children were generally more likely to be in problematic relationship trajectories characterised by stable-low child-teacher warmth and increases in child-teacher conflict throughout elementary school [6].

\section{The Present Study}

There is a need to develop further understanding as to why some groups of children follow atypical maladaptive child-teacher relationship trajectories, when others follow more normative positive relationship trajectories [6]. The goal of the present study was to a) describe person-centered child-teacher relationship trajectories experienced by young children between preschool (age 4) and the first and second years of formal schooling (age 5 and 6); and b) identify characteristics of children, families and their contextual relationships that were associated with different child-teacher relationship trajectories during this period of children's development.

A secondary aim of the present study was to examine predictors of child-teacher relationship trajectories for children considered psychologically at-risk at preschool due to exhibiting early mental health problems, so that any potential protective factors could be identified that may help these children especially at-risk for poor quality relationships to build better child-teacher relationships [5].

The present study builds upon previous research on correlates of child-teacher relationship quality trajectories [5], [24], by examining predictors of trajectories across the transition from preschool into formal schooling, a time considered important given the critical transition into a formal school environment, and given that relationships with first teachers seem to form a basis for future relationships [5], [6]. This study also extends previous research by examining the effect of several potential socio-demographic predictors of child-teacher relationship trajectories (e.g., prosocial skills, parenting styles, parental distress), which have so far remained unexplored. According to [22], the present study also represents a much-needed extension upon existing research by being one of only a small number of studies in this field to be conducted outside of the United States.

Given the dynamic nature of potential relationships between children and the different teachers they have during preschool and school, we hypothesized that there would be distinct subgroups of children who would follow different trajectories of relationship quality over time. For example, while some children may have positive relationships with each of their teachers over time, there may be an identifiable group of children who show low or declining levels of relationship quality over time. We also hypothesized that experiencing more problematic child-teacher relationship trajectories would be predicted by children having early mental health difficulties ,being male, having a disability, less prosocial behavior skills, having family characteristics indicative of socio-economic disadvantage and family stress, maladaptive parenting techniques, fewer interactions with teachers, and poorer quality parent-teacher relationships.

\section{METHOD}

\section{A. Participants and Procedure}

This three-year longitudinal study focused on 636 children who were part of a larger study on child development. Initially, 700 children attending the 27 government-funded preschools in one South Australian Government schooling district participated at baseline. While the demographic characteristics of the participating district overall resemble those for South Australia as a whole, it is quite diverse as it encompasses suburban, rural, and remote regions, and some of the highest and lowest ranked areas of socio-economic disadvantage in Australia[27].

Participation was sought from families of all children attending preschools within the district. Preschools in South Australia provide a government-funded education program available for up to 15 hours a week to all 4 year-old children for 12 months prior their commencing formal schooling. Over $90 \%$ of children attend preschool in South Australia [28].

Data collection for the baseline assessment was coordinated by the director at each preschool (with assistance from the research team). Distribution and retrieval of confidential parent-completed questionnaires was arranged through the preschools. Teacher-reported questionnaires were completed by the teacher who "best knows the child". Informed consent was obtained from parents prior to the completion of parent and teacher questionnaires.

At baseline, teacher-reports (from 57 different teachers) were obtained for 700 children (representing $72.4 \%$ of all district preschool children). For 601 of these 700 children, a parent survey was also completed $(62.2 \%$ of all district preschool children). A total of $51 \%$ of participating children were female, and the average age was 4.66 years (55.9 months, $S D=3.9)$. Based on school district records, the participating children were of similar age and gender distribution to the preschool children in the whole district, but Indigenous children were under-represented.

Two follow-up assessments were conducted 12 months later and 24 months after the baseline assessment, by which time children had commenced their formal schooling. At the time of these assessments, parent-completed questionnaires were mailed to family homes, and returned to the researchers in reply-paid envelopes. Children were followed regardless of their school destination, but the majority (69\%) were attending government schools in the same district as their preschool. A nominated liaison person at each school helped the research team distribute and collect the second and third teacher-rated assessments. In order to allow time to get to know any newly commencing students, teachers were required to have interacted with them for a minimum of 5 weeks before completing surveys about the child. At each assessment, parent-rated surveys and teacher-rated surveys took approximately 30 and 10 minutes to complete, respectively.

All three assessments requested from teachers were completed for 642 of the participating children $(92.7 \%$ 
retention rate), and 636 had complete data on all 3 child-teacher relationship questionnaires. The baseline parent-reported surveys $(n=601)$ were completed predominantly by mothers $(92 \%)$.In the first column of results in Table I, the response sample $n$ and descriptive statistics for the current study variables are provided.

The study methodology was approved by the Research Ethics Committees at the Women's and Children's Hospital Adelaide, and the South Australian Department of Education and Children's Services. More detailed information on the study methodology can be found in [9].

\section{B. Measures}

Children's current teacher and their primary care-giving parent completed the following standardized questionnaires at each yearly assessment.

Child-teacher relationship quality was measured at each time-point with the Student-Teacher Relationship Scale Short Form [29], [30]. Teachers rated their perceptions of their relationship with the child on 15 items using a 5-point Likert scale ("definitely does not apply" to "definitely applies"). Items assess relationship conflict, closeness, and warmth (e.g., "If upset, this child will seek comfort from me"). A total score (possible range $=15-75$ ), is created by summing the 15 items, with higher scores indicating a better quality relationship. The STRS has good psychometric properties [30].

The Strengths and Difficulties Questionnaire (SDQ) [31], was completed at each assessment by the child's primary-care giving parent and their current teacher. The SDQ is a 25-item psychiatric screening questionnaire for children. The items represent five subscales: Emotional Symptoms; Conduct Problems; Hyperactivity; Peer Problems; and Prosocial Behavior. Respondents provide answers ("not true", "somewhat true", or "certainly true") about the child's behavior over the previous six months or the current school year. Scores on each subscale can range from 0 to 10 . A Total Difficulties score is generated by summing the subscale scores, with the exception of the prosocial subscale (for which higher scores indicate better prosocial behavior skills). Scores on Total Difficulties can range from 0 to 40, with higher scores indicating greater mental health difficulties. Scores exceeding 14 on parents reports and 12 on teachers reports indicate children in the borderline clinical 'of concern' range. The SDQ has strong psychometric properties [32].

Children's socioeconomic and demographic background was assessed during the baseline assessment. Whether the child was living in a two-parent family or a single-parent family was determined by which parental figures currently lived with the child. The responding parent was asked to indicate whether or not the family received government welfare benefits for lower-income families, and whether the child had a serious illness or a disability. Responses to questions on the level of full-time equivalent employment of both the mother and the father were used to determine the combined level of parental employment, which could range from none to two full-time jobs. The responding parent also reported on the mothers' and the fathers' highest level of completed educational qualifications. The primary care giving parent also reported on their level of psychological distress on the 12-item version of the General Health Questionnaire [33], and the number of stressful life events the family had experienced in the previous 12 months (using a modified 12-item version of the List of Threatening Life Experiences Questionnaire) [34]. The primary care-giving parent also reported on the level of warmth in their relationship with the child (using the warmth subscale of the Child Rearing Practices Scale) [35], and on their use of hostile-ineffective parenting strategies(on a 7-item scale from the Canadian National Longitudinal Survey of Children and Youth) [36].The parent also reported on the quality of their relationship with their child's preschool teacher(s) (the 'relationship with caregiver' subscale of the NICHD Parent-Caregiver Involvement Questionnaire) [29].Finally, school records at the third assessment were used to calculate the number of school terms the child had been attending their school, and whether or not they had spent more than 12 months in class with the same teacher since starting school.

\section{Statistical Analyses}

Analyses were conducted in two stages. First, we assessed whether there were distinct subgroups of children who followed different trajectories of child-teacher relationship quality over the preschool-to-school transition. This was achieved using Latent Class Growth Modeling (performed with PROC TRAJ in SAS), which is a semi-parametric group-based modeling approach that estimates individual growth curves for each child and then identifies subgroups of children who follow the same trajectory over time [37]-[38].This analysis utilized the full sample with complete data on STRS at the 3 assessments $(n=636)$, in order to provide the best estimate of trajectories of student teacher relationship quality. To identify the best fitting number of trajectories we fit a series of models using the following criteria:1) whether the trajectory shape was statistically $\operatorname{significant}(p<.05)$; 2) whether the Bayesian Information Criteria (BIC) value for models with increasing numbers of groups showed evidence of improved fit; 3 ) whether at least $5 \%$ of the sample were identified as belonging to each trajectory; and 4) whether the probability of group membership for each trajectory was 0.70 or higher, indicating that the identified trajectories were indeed grouping together individuals with similar patterns of change over time [38], [39].

The second set of analyses used logistic regression to examine the strength of the association between child, family, and demographic characteristics when the children were in preschool, and child-teacher relationship trajectories between preschool and the second year of school. First, we examined the strength of the bivariable relationship between each predictor variable and child-teacher relationship trajectory groups. Second, simultaneous multiple logistic regression analyses containing all the predictor variables examined the strength of this relationship while adjusting for the effects of all the other predictor variables. Scores on continuous predictor variables were standardised prior to statistical analysis to enable comparison of the predictive ability of variables. Thus, the resulting odds ratio $(O R)$ demonstrated the change in risk of target group membership for every unit of change in standard deviation $(S D)$ in the predictor variable. These logistic regressions were conducted for the whole sample combined, and then separately for children who were (and were not) deemed psychologically at-risk based on scoring above the borderline-clinical cut-off on teacher-rated 
SDQ mental health difficulties at baseline.

\section{RESULTS}

\section{A. Trajectories of Child-Teacher Relationship Quality}

Moderate positive correlations were found between the STRS scores on child-teacher relationship quality reported by teachers across the three assessments (Time 1 correlated with Time 2 at $r=0.41, p<.001$; Time 1 correlated with Time 3 at $r=0.27, p<.001$; and Time 2 correlated with Time 3 at $r=0.49$, $p<.001)$. The STRS scores were positively skewed at each of the assessment time points, with the majority of teachers reporting positive relationships with their students (theinterquartile ranges were $41-75$ at Time 1, 36-75 at Time 2, and 38-75 at Time 3). On average for the whole sample, STRS scores decreased over time - with means (SDs) of 67.2 (8.5) at Time 1, 66.6 (8.8) at Time 2, and $65.9(9.2)$ at Time 3.

Child-teacher relationship trajectories were found to be linear in shape across the three assessments. The BIC value for one linear trajectory was -6244 , but improved fit was indicated for two linear trajectories with a BIC value of -6103.03. The estimate of the log Bayes factor was calculated in order to further examine this evidence of improved fit [40]. The log Bayes factor estimate was 276.5, providing strong evidence for improved model fit [40]. Because the third trajectory included less than $5 \%$ of the sample, there was no evidence for a three-trajectory model. For the two linear trajectory model, the probability of group membership was 0.89 for trajectory 1 , and 0.98 for trajectory 2 . This suggested that the two identified trajectories grouped individuals with similar patterns of change over time. The two linear trajectory model identified one statistically significant trajectory $(p<.001)$ and a second trajectory that approached statistical significance $(p=.08)$. The two linear trajectory model was selected as the final model, because of the strong evidence of good model fit and existence of two distinct trajectories.

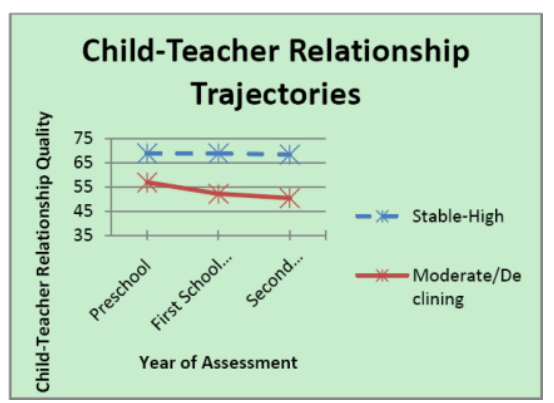

Fig. 1. Identified trajectories of child-teacher relationship quality.

Fig. 1 shows the two trajectories identified, which were labeled (a) 'Stable High Relationship Quality', and (b) 'Moderate/Declining Relationship Quality'. The Stable High trajectory group ( $n=550,86.5 \%$ of the sample) had high quality child-teacher relationships at preschool $(M=68.9$, $S D=6.3)$, and in the first $(M=68.9, S D=5.9)$, and second $(M=68.4, S D=6.3)$ years of school. The Moderate/Declining trajectory group $(n=86,13.5 \%$ of the sample $)$ had child-teacher relationships of moderate quality at preschool $(M=56.9, S D=11.9)$, which then declined in the first year of school $(M=52.2, S D=10.1)$, and declined again in the second year of school $(M=50.4, S D=9.5)$.

\section{B. Predictors of Child-Teacher Relationship Quality Trajectories}

Table I (under the subheading 'whole sample logistic regressions') shows the results of the bivariable (unadjusted) logistic regression analyses assessing strength of the association of each predictor variable with child-teacher relationship trajectory group. In these analyses, the stable-high trajectory group was treated as the reference group (coded 0), and the Moderate/Declining child-teacher relationship quality trajectory group was the target group (coded 1). It can be seen that the majority of the predictor variables held significant unadjusted associations with trajectory group membership. Children scoring above the borderline-clinical cut-off on preschool teacher-reported SDQ total mental health difficulties were 10.5 times more likely to show a moderate/declining child-teacher relationship trajectory $(O R=10.5,95 \% \quad C I=6.3-17.5)$. Similarly, children scoring above the same cut-off on parent-reports were 2.1 times more likely to be in the moderate/declining child-teacher relationship trajectory group than the stable-high child-teacher relationship trajectory group $(O R=2.1,95 \% C I=1.2-3.9)$. Children were also more likely to belong to the moderate/declining child-teacher relationship trajectory group if they were from a single parent family, a family receiving a government welfare benefit, a family experiencing more stressful life events, and were exposed to more hostile/ineffective parenting techniques. Children were less likely to belong to the moderate/declining child-teacher relationship trajectory group if they were female, had parents with a higher level of employment, a mother with higher educational qualifications, had better prosocial skills at preschool (according to both teacher and parent), had a warmer relationship with their primary caregiving parent, had spent more time at their current school, and had parents who held a better quality relationship with the child's preschool teachers.

The multivariable logistic regression model for the whole sample was statistically significant, $\chi^{2}(d f=19)=97.06, p$ $<.001$, indicating that these 19 predictors together detected systematic differences between children exhibiting a moderate/declining child-teacher relationship trajectory versus children exhibiting a high-stable child-teacher relationship trajectory. Overall, the predictors together accounted for approximately $34.1 \%$ of the variation in child-teacher relationship trajectories (Nagelkerke $R^{2}$ estimate $=0.341$ ). The adjusted $O R$ ' $s$ in Table I (under the subheading 'whole sample logistic regressions') indicate that four predictor variable scores were significantly associated with belonging to the moderate/declining child-teacher relationship trajectory after adjusting for all other predictor variables in the analysis. Having teacher-reported SDQ mental health problems above the cut-off at preschool remained a highly significant predictor of child-teacher relationship trajectories $(O R=4.8,95 \% C I=1.9-11.7)$. Greater hostile-ineffective parenting also remained a significant risk factor for having a moderate-declining child-teacher relationship trajectory, and being from a single-parent family approached significance as a predictor in the adjusted model. Being female and having more teacher-rated prosocial skills at preschool resulted in being significantly less likely to 
havea moderate-declining relationship trajectory, after controlling for all other variables in the model.

TABLE I: DESCRIPTIVE STATISTICS AND LOGISTIC REgRESSIONS PREDICTING MEMBERSHIP TO THE MODERATE-DECLINING CHILD-TEACHER RELATIONSHIP TRAJECTORY GROUP FOR CHILDREN AT-RISKAND NOT AT-RISK (ODDS RATIOS) ${ }^{\mathrm{A}, \mathrm{B}, \mathrm{C}}$

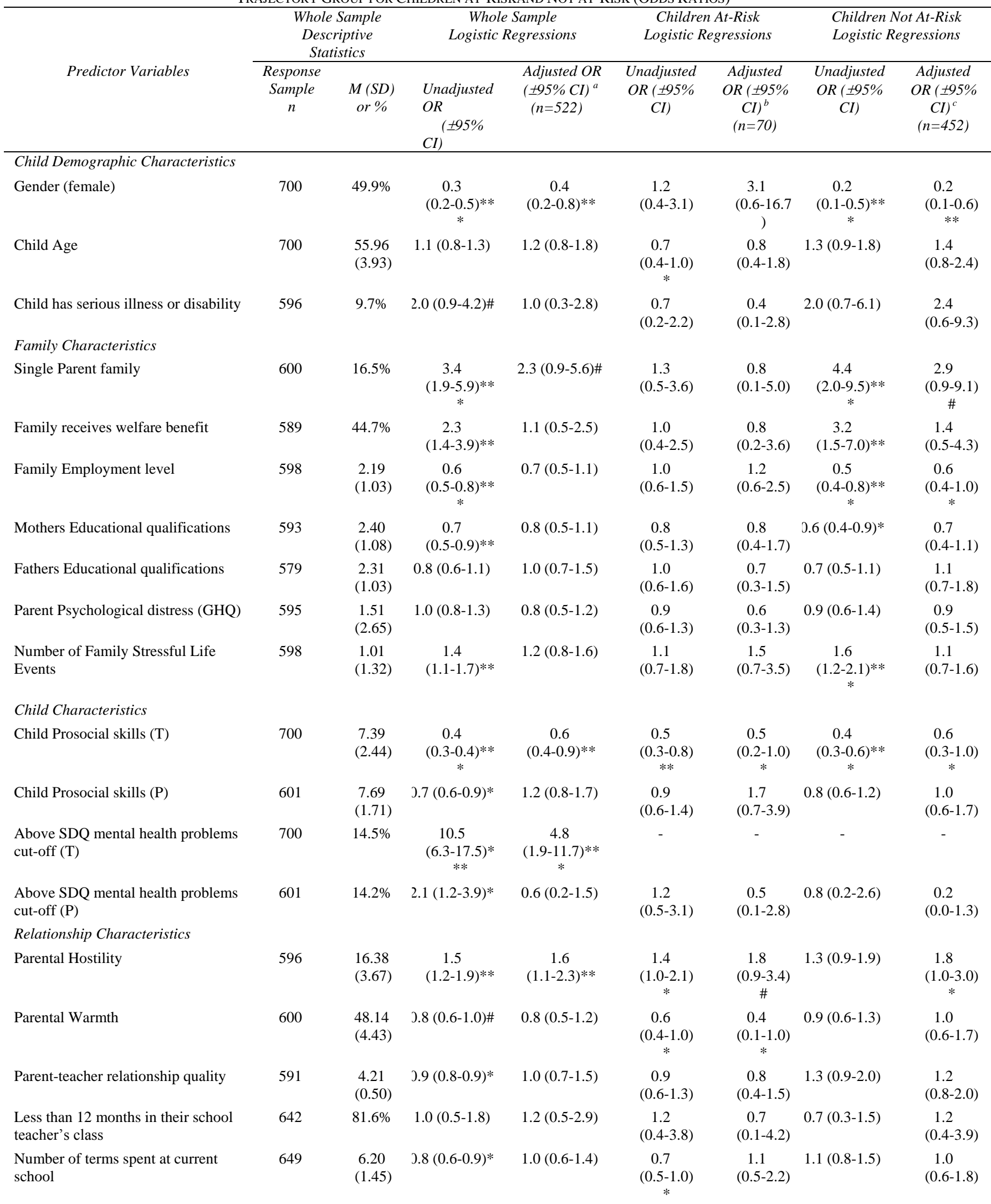

Notes.\# $p<.10 ; * p<.05 ; * * p<.01 ; * * * p<.001 . O R=$ Odds Ratio; $C I=$ Confidence Interval; $P=$ Parent-reported; $T=$ Teacher-reported; Unadjusted models have different

$n$ 's depending on the missing data. $n$ 's range from 553 to 636 for the analyses using the whole sample; $n$ 's range from 473 to 542 for the children not at-risk; $n$ 's range from 73 to 94 for children at-risk.

a Final Model for whole sample: $\chi^{2}(\mathrm{df}=19)=97.06^{* * *}{ }^{\text {b }}$. Cox \& Snell $R^{2}$ estimate $=0.170 ;$ Nagelkerke $R^{2}$ estimate $=0.341$.

b. Final Model for children at-risk: $\chi^{2}(\mathrm{df}=18)=22.49 ;$ Cox \& Snell $R^{2}$ estimate $=0.275 ;$ Nagelkerke $R^{2}$ estimate $=0.370$.

'. Final Model for children not at-risk: $\chi^{2}(\mathrm{df}=18)=49.19^{* * *} ;$ Cox \& Snell $R^{2}$ estimate $=0.103 ;$ Nagelkerke $R^{2}$ estimate $=0.278$. 


\section{Predictors of Child-Teacher Relationship Quality}

Trajectories for Children Psychologically at-Risk and Not at-Risk

Given the strong association evident between child-teacher relationship trajectories and preschool teacher-rated SDQ mental health difficulties, we repeated the logistic regression analyses separately for children who could be considered psychologically at-risk due to the presence of mental health problems during preschool. Such an analysis may help to understand what predictors are particularly important for children experiencing early mental health problems identified by teachers in preschool, in comparison to children not identified as experiencing these problems. Of the 700 children at baseline, $102(14.6 \%)$ were classified as at-risk due to mental health problems, and 598 (85.4\%) were considered not-at-risk.

The results of the bivariable logistic regression analyses assessing the strength of the association between each predictor variable and the child-teacher relationship trajectory group for the at-risk children are shown in Table I (under the subheading 'children at-risk logistic regressions'). At-risk children were significantly more likely to belong to the moderate/declining child-teacher relationship trajectory group if they experienced more hostile/ineffective parenting techniques, and were significantly less likely to be in the moderate/declining child-teacher relationship trajectory group if they were older, had better prosocial skills at preschool (according to their teacher), had a warmer relationship with their primary care giving parent, and had spent more time at their current school. In contrast, the unadjusted predictors for children not-at-risk (based on preschool SDQ difficulties below the cut-off), were more numerous and more closely resembled those found for the whole sample (see Table I under the subheading 'children not-at-risk logistic regressions').Children with family characteristics indicative of stress and socioeconomic disadvantage were more likely to be in the moderate/declining trajectory group. Girls and children with more prosocial skills were less likely to belong to the moderate/declining trajectory group.

The multivariable logistic regression models for children psychologically at-risk and those not-at-risk overall indicated greater success in identifying unique predictors of child-teacher relationship trajectories in the children not-at-risk (see model fit at the bottom of Table I). For children deemed psychologically at-risk, two predictors were significantly associated with belonging to the moderate/declining child-teacher relationship trajectory after adjusting for all other predictor variables in the analysis. At-risk children with more teacher-rated prosocial skills at preschool were significantly less likely to have a moderate-declining relationship trajectory, as were those experiencing greater parental warmth. Greater hostile-ineffective parenting also approached significance as a predictor for at-risk children having a moderate-declining child-teacher relationship trajectory, after controlling for all other variables in the model. For children deemed not-at-risk, four predictors were significantly associated with belonging to the moderate/declining child-teacher relationship trajectory after adjusting for all other predictor variables. Similar to at-risk children, those not-at-risk psychologically who had better teacher-rated prosocial skills at preschool were significantly less likely to have a moderate-declining relationship trajectory, and those exposed to greater hostile-ineffective parenting were significantly more likely to belong to the moderate-declining child-teacher relationship trajectory. Also, children not-at-risk who were female and had parents with a greater level of employment were significantly less likely to be in the moderate/declining relationship trajectory, after controlling for all other variables in the model.

\section{Discussion}

This longitudinal study represents one of only a handful of studies to examine predictors of trajectories in child-teacher relationship quality. Using the person-centered trajectory method of latent class growth modeling, we identified two subgroups: a stable high quality child-teacher relationship trajectory, and a more atypical moderate/declining quality child-teacher relationship trajectory, a pattern which characterized $13.5 \%$ of our sample. The trajectory patterns found in the present study resembled those found by [10], who also identified a subgroup of $13 \%$ that had poor-declining child-teacher relationships.

Consistent with previous research [5], [20], [24], we found that the strongest predictor of belonging to the moderate/declining child-teacher relationship trajectory was the presence of clinically significant teacher-rated mental health difficulties during preschool. It is possible that strained interactions between preschool teachers and children with early mental health problems (which more frequently require re-direction, intervention, and behavior management), may set these children on a pathway to persisting negative relationships with teachers.

Children exposed to a hostile-ineffective parenting style were also more likely to belong to this poorer quality child-teacher relationship trajectory, whereas girls and children with more prosocial behavior skills at preschool were less likely to have a moderate/declining trajectory. These findings add further evidence corroborating previous research [5], [7], [13], [24]. Whilst factors indicative of socioeconomic disadvantage were more common for children in the moderate/declining trajectory, these factors tended to only significantly influence trajectory outcomes when considered alone - once other factors such as parenting and child skills were adjusted for, their effects frequently disappeared. This suggests that the influence of socioeconomic disadvantage on child-teacher relationships may be mediated by these other factors.

Prosocial behavior skills in preschool were a robust predictor of healthy stable-high quality child-teacher relationship trajectories, being significant in unadjusted and adjusted analyses, and for psychologically at-risk children, not-at-risk children, and for the whole sample. Therefore prosocial skills were important for all children, as well as being especially protective for children considered psychologically at-risk at preschool due to exhibiting early mental health problems. Parental warmth also appeared to protect these children against experiencing a moderate/declining child-teacher relationship trajectory.

The following limitations need to be considered when 
interpreting the results of this study. First, only the teacher was used as an informant on child-teacher relationship quality. Thus, it is not known how biased teachers reports were by their perceptions. Obtaining additional reports of relationship quality from children and using direct observations would bolster construct validity and reduce common-method variance. Second, the associations with child-teacher trajectories were much stronger for preschool teacher-reports of mental health difficulties and prosocial behavior, than for parent-reports on these characteristics. It is possible that common-reporter bias on preschool SDQ and the preschool component of child-teacher relationship trajectories may have inflated these associations, even though the second and third assessments of child-teacher relationships were reported by different teachers. Future research could consider assessing these factors at multiple time-points during preschool. This might highlight the transactional processes within these relationships, whereby children with poor mental health may have more barriers to forming positive relationships with teachers, which in turn may lead to more mental health problems, and so on [23]. Third, we lacked information on characteristics of teachers, classes, and schools (e.g., class size; teacher qualifications) that may have held associations with child-teacher relationship trajectories, and may have changed the pattern of results if included in the adjusted models. Finally, it is unclear whether within-school clustering effects influenced our results.

\section{CONCLUSIONS AND IMPLICATIONS}

In conclusion, the predictors of poor quality child-teacher relationship trajectories found in this study appear to be factors that impact on the child's ability to form relationships (prosocial skills, mental health problems), and their positive and negative experiences of relationships (hostile parenting and parental warmth). Where poor child-teacher relationships exist, early interventions to address these risk factors may be important to improve the child's ability to successfully form positive relationships and also increase their experience of having supportive positive relationships.

Given the implications of poorer quality child-teacher relationship trajectories for children's development [6], [25], helping children develop high quality relationships is vital.The findings of the present study help us to understand the characteristics of children who are more likely to experience cumulatively poorer quality teacher relationship trajectories. Given children with early mental health problems are especially at-risk, targeted interventions designed to foster positive interactions between teachers and these children may be worthwhile. The robust influence of prosocial behavior skills in preschool as a predictor of healthy stable-high quality child-teacher relationship trajectories in at-risk and not-at-risk children implies that this may form a viable skill set to target for improvement using preschool-based early-intervention and prevention efforts.

It is likely to be more difficult for teachers to form positive relationships with children facing these risk factors, but teachers are uniquely placed to help children improve these emotional, behavioral and social skills. Teachers are also well-placed to provide children with the opportunity to experience a positive relationship with a supportive adult, which is fundamentally important for children developmentally [3], [4], [9], [21], [25]. It is possible that having a positive relationship with an adult such as a teacher might help to reorganize relational models and promote better outcomes for children at-risk due to their experience of more hostile-ineffectiveparenting and less parental warmth [22].

Knowledge on predictors of poor quality child-teacher relationships may be useful to impart in teacher training and professional development. Teaching educators about the effects of child characteristics on the quality of child-teacher relationships may help to prevent teachers from developing poorer quality relationships with children on the basis of those particular characteristics [5]. For example, knowing that boys and children from single parent families, and children who struggle socially are at greater risk for poor quality child-teacher relationships, may make teachers more mindful of counteracting this possibility by adjusting the frequency and type of their interactions with these children. These strategies, embedded within professional development interventions which have been shown to improve child-teacher interactions (such as 'banking time' with children) [22], may help place children on more positive developmental trajectories.

\section{ACKNOWLEDGMENT}

We would like to acknowledge the contributions of Mike Hudson, Debra Kay, Richard Costi, Megan Tassell, Jenny Boag, Tania Liston, Sue Klar, Jillian Jordan, and other members of our Research Project Advisory Team from the South Australian Department of Education and Child Development. We would also like to thank the teachers and families who participated in this research. Finally, we are grateful to John Lynch, Murthy Mittinty, and Nancy Briggs for their research advice and statistical assistance.

\section{REFERENCES}

[1] B. K. Hamre and R. C. Pianta, "Early teacher-child relationships and the trajectory of children's school outcomes through eighth grade," Child Development, vol. 72, pp. 625-638, 2001

[2] S. H. Birch and G. W. Ladd, "The teacher-child relationship and children's early school adjustment," Journal of School Psychology, vol. 35, pp. 61-79, 1997.

[3] S. S. Myers and R. C. Pianta, "Developmental commentary: Individual and contextual influences on student-teacher relationships and children's early problem behaviors," Journal of Clinical Child and Adolescent Psychology, vol. 37, pp. 600-608, 2008.

[4] A. J. Mashburn and R. C. Pianta, "Social relationships and school readiness," Early Education \& Development, vol. 17, pp. 151-176, 2006.

[5] E. O'Connor, "Teacher-child relationships as dynamic systems," Journal of School Psychology, vol. 48, pp. 187-218, 2010

[6] J. L. Spilt, J. N. Hughes, J.-Y. Wu, and O.-M. Kwok, "Dynamics of teacher-student relationships: Stability and change across elementary school and the influence on children's academic success," Child Development, vol. 83, pp. 1180-1195, 2012.

[7] C. Howes, "Social-emotional classroom climate in child care, child-teacher relationships and children's second grade peer relations," Social Development, vol. 9, pp. 191-204, 2000.

[8] J. Hughes and O. M. Kwok, "Influence of student-teacher and parent-teacher relationships on lower achieving readers' engagement and achievement in the primary grades," Journal of Educational Psychology, vol. 99, pp. 39-51, 2007.

[9] L. R. Miller-Lewis, A. K. Searle, M. G. Sawyer, P. A. Baghurst, and D. Hedley, "Resource factors for mental health resilience in early childhood: an analysis with multiple methodologies," Child and Adolescent Psychiatry and Mental Health, vol. 7, pp. 6, 2013. 
[10] E. O'Connor and K. McCartney, "Examining teacher-child relationships and achievement as part of an ecological model of development," American Educational Research Journal, vol. 44, pp. 340-369, 2007.

[11] A. K. Searle, L. R. Miller-Lewis, M. G. Sawyer, and P. A. Baghurst, "Predictors of children's kindergarten classroom engagement: Preschool adult-child relationships, self-concept, and hyperactivity/inattention," Early Education and Development, vol. 24 pp. 1112-1136, 2013.

[12] A. S. Eisenhower, B. L. Baker, and J. Blacher, "Early student-teacher relationships of children with and without intellectual disability: Contributions of behavioral, social, and self-regulatory competence,' Journal of School Psychology, vol. 45, pp. 363-383, 2007.

[13] C. S. Chan, J. E. Rhodes, W. J. Howard, S. R. Lowe, S. E. O. Schwartz, and C. Herrera, "Pathways of influence in school-based mentoring: The mediating role of parent and teacher relationships," Journal of School Psychology, vol. 51, pp. 129-142, 2013

[14] E. O'Connor and K. McCartney, "Testing associations between young children's relationships with mothers and teachers," Journal of Educational Psychology, vol. 98, pp. 87-98, 2006.

[15] R. C. Pianta, S. L. Nimetz, and E. Bennett, "Mother-child relationships, teacher-child relationships, and school outcomes in preschool and kindergarten," Early Childhood Research Quarterly, vol. 12, pp. 263-280, 1997.

[16] P. Mantzicopoulos, "Conflictual relationships between kindergarten children and their teachers: Associations with child and classroom context variables," Journal of School Psychology, vol. 43, pp. 425-442, 2005.

[17] G. W. Ladd, S. H. Birch, and E. S. Buhs, "Children's social and scholastic lives in kindergarten: related spheres of influence?" Child Development, vol. 70, pp. 1373-1400, 1999.

[18] G. W. Ladd and K. B. Burgess, "Do relational risks and protective factors moderate the linkages between childhood aggression and early psychological and school adjustment?" Child Development, vol. 72, pp. $1579-1601,2001$

[19] R. C. Pianta, K. M. la Paro, C. Payne, M. J. Cox, and R. Bradley, "The relation of kindergarten classroom environment to teacher, family, and school characteristics and child outcomes," The Elementary School Journal, vol. 102, pp. 225-238, 2002.

[20] J.-E. Nurmi, "Students' characteristics and teacher-child relationships in instruction: A meta-analysis," Educational Research Review, vol. 7 , pp. 177-197, 2012.

[21] B. T. Meehan, J. N. Hughes, and T. A. Cavell, "Teacher-student relationships as compensatory resources for aggressive children," Child Development, vol. 74, pp. 1145-1157, 2003.

[22] T. J. Sabol and R. C. Pianta, "Recent trends in research on teacher-child relationships," Attachment \& Human Development, vol. 14, pp 213-231, 2012.

[23] S. Doumen, K. Verschueren, E. Buyse, V. Germeijs, K. Luyckx, and B. Soenens, "Reciprocal relations between teacher-child conflict and aggressive behavior in kindergarten: A three-wave longitudinal study," Journal of Clinical Child and Adolescent Psychology, vol. 37, pp. $588-599,2008$

[24] E. M. Jerome, B. K. Hamre, and R. C. Pianta, "Teacher-child relationships from kindergarten to sixth grade: Early childhood predictors of teacher-perceived conflict and closeness," Social Development, vol. 18, pp. 915-945, 2009.

[25] E. E. O'Connor, B. A. Collins, and L. Supplee, "Behavior problems in late childhood: The roles of early maternal attachment and teacher-child relationship trajectories," Attachment \& Human Development, vol. 14, pp. 265-288, 2012.

[26] E. E. O'Connor, E. Dearing, and B. A. Collins, "Teacher-child relationship and behavior problem trajectories in elementary school,' American Educational Research Journal, vol. 48, pp. 120-162, 2011.

[27] Australian Bureau of Statistics, Catalogue 4442.0: Family Characteristics Australia, 2003, Commonwealth of Australia, Canberra, 2004.

[28] Steering Committee for the Review of Government Service Provision, Report on Government Services 2008: Children's Services, Productivity Commission, Canberra, 2008.

[29] NICHD Early Child Care Research Network. (2000). NICHD study of Early Child Care and Youth Development: Phase 2 Instrument Document. [Online]. http://secc.rti.org/Phase2InstrumentDoc.pdf

[30] R. C. Pianta, Student-Teacher Relationship Scale, Florida: Psychological Assessment Resources Inc., 2001.

[31] R. Goodman, "The Strengths and difficulties questionnaire: A research note," Journal of Child Psychology and Psychiatry, vol. 38, pp. 581-586, 1997
[32] R. Goodman, "Psychometric properties of the strengths and difficulties questionnaire," Journal of the American Academy of Child and Adolescent Psychiatry, vol. 40, pp. 1337-1345, 2001

[33] D. P. Goldberg and P. Williams, A User's Guide to the General Health Questionnaire, Windsor: NFER-Nelson, 1991.

[34] T. Brugha, P. Bebbington, C. Tennant, and J. Hurry, "The List of Threatening Experiences: A subset of 12 life event categories with considerable long-term contextual threat," Psychological Medicine, vol. 15, pp. 189-194, 1985.

[35] A. Sanson, Child Rearing Questionnaire, Unpublished Manual: University of Melbourne, Australia, 1996.

[36] Statistics Canada. (1995). National Longitudinal Survey of Children: Survey Instruments for 1994-95 Data Collection, Cycle 1 [Electronic Version]. Catalogue No. 89F0077XIE. [Online]. Available: http://www.statcan.gc.ca/pub/89f0077x/89f0077x1996001-eng.pdf.

[37] B. L. Jones and D. S. Nagin, "Advances in group-based trajectory modeling and an SAS procedure for estimating them," Sociological Methods \& Research, vol. 35, pp. 542-571, 2007.

[38] D. S. Nagin, "Analyzing developmental trajectories: A semiparametric, group-based approach," Psychological Methods, vol. 4, pp. 139-157, 1999.

[39] H. Andruff, N. Carraro, A. Thompson, P. Gaudreau, and B. Louvet, "Latent class growth modelling: A tutorial," Tutorials in Quantitative Methods for Psychology, vol. 5, pp. 11-24, 2009.

[40] B. L. Jones, D. S. Nagin, and K. Roeder, "A SAS procedure based on mixture models for estimating developmental trajectories," Sociological Methods \& Research, vol. 29, pp. 374-393, 2001.

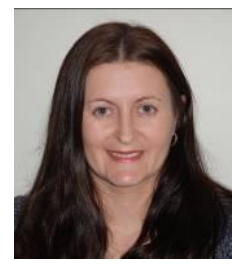

Lauren R. Miller-Lewis was born in Australia. She obtained a Bachelor of Psychology (with $1^{\text {st }}$ class Honours) and a PhD in Psychology from Flinders University in Adelaide, South Australia, in 2001 and 2004, respectively. She is currently a Public Health Post-Doctoral Research Training Fellow for the NHMRC (National Health and Medical Research Council), co-located at the Discipline of Paediatrics, School of Paediatrics and Reproductive Health at the University of Adelaide, and the Research and Evaluation Unit at Adelaide's Women's and Children's Hospital. Her research interests broadly encompass developmental and educational psychology, with a focus on the mental health, wellbeing, and resilience of children in the early years of education. Dr. Miller-Lewis is a member of the Australian Psychological Society (APS), the Australian Research Alliance for Children and Youth, and Healthy Development Adelaide.

Alyssa C. P. Sawyer was born in Australia. She obtained a Graduate Diploma and $1^{\text {st }}$ class Honours in Psychology in 2006 and 2007 from Flinders University. She completed a PhD in Clinical Psychology at Flinders University in 2012. Dr. Sawyer is currently a Post-Doctoral Research Fellow in the Discipline of Public Health, School of Population Health at the University of Adelaide. She also works as a registered psychologist in private practice with children, adolescents, and their families. Dr. Sawyer is a Member of the APS and Associate Member of the APS Clinical College, Member of the Australian Association for Cognitive Behavioural Therapy, and Healthy Development Adelaide.

Amelia K. Searle was born in Australia. She obtained a Bachelor of Psychology (with $1^{\text {st }}$ class Honours) from Flinders University in 2004, and a $\mathrm{PhD}$ in Paediatrics and Psychology from the University of Adelaide in 2011 She is currently a Senior Research Officer at the Centre for Traumatic Stress Studies in the School of Population Health at the University of Adelaide. Dr. Searle is a member of the Australasian Human Development Association, the Australian Research Alliance for Children and Youth, the Australasian Society for Traumatic Stress Studies, and Healthy Development Adelaide.

Michael G. Sawyer was born in England. He obtained a Bachelor of Medicine (MBBS) in 1972 from Monash University in Australia, a Diploma of Child Psychiatry in 1980 from the University of Toronto, Canada, and a $\mathrm{PhD}$ in Child Psychiatry from the University of Adelaide in 1991. Professor Sawyer is the Head of the Research and Evaluation Unit at Adelaide's Women's and Children's Hospital, and Professor of Child and Adolescent Psychiatry in the Discipline of Paediatrics, School of Paediatrics and Reproductive Health at the University of Adelaide. Professor Sawyer is a fellow of the Royal Australian and New Zealand College of Psychiatrists and of the Royal College of Physicians and Surgeons of Canada, and he is Co-Chair of Healthy Development Adelaide. In 2008, Professor Sawyer was awarded a Medal of the Order of Australia for services to the field of child and adolescent mental health. 